\title{
Health-related quality of life in parents of children with intermittent exotropia
}

\author{
Tomohiko Yamada, OD, Sarah R. Hatt, DBO, David A. Leske, MS, and Jonathan M. Holmes, \\ $\mathrm{BM}, \mathrm{BCh}$ \\ Department of Ophthalmology, Mayo Clinic, Rochester, Minnesota
}

\begin{abstract}
Purpose-To assess the health-related quality of life (HRQOL) of parents of children with intermittent exotropia using the newly developed condition-specific Parent Intermittent Exotropia Questionnaire (Parent IXTQ) and the generic PedsQL Family Impact Module (PedsQL FIM) and to compare the performance of both instruments.
\end{abstract}

\begin{abstract}
Methods-One parent was recruited for each of 59 children with intermittent exotropia (age range, 3 to 16; median, 7 years) and for each of 29 visually normal children (age range, 5 to 13; median, 8 years). The parent completed the Parent IXTQ and the PedsQL FIM. For each questionnaire, we compared median HRQOL scores between the intermittent exotropia group and visually normal group. We also calculated normal thresholds, which were defined as the 5th percentile score in the cohort of parents of visually normal children. The proportion of subnormal scores between questionnaires in parents of children with intermittent exotropia was compared.
\end{abstract}

Results-The Parent IXTQ score was worse in the intermittent exotropia group than in the visually normal group (70.6 vs 94.1, $p<0.0001$ ), whereas the PedsQL FIM scores were similar (97.9 vs 95.8, $p=0.8$ ). More parents scored below normal using the Parent IXTQ than using the PedsQL FIM ( $31 \%$ vs $12 \%$; $p=0.008$, McNemar's test).

Conclusions-The Parent IXTQ detects subnormal HRQOL in parents of children with intermittent exotropia more often than the PedsQL FIM. It is possible that parental worry may influence management decisions in children with intermittent exotropia and therefore parental HRQOL is worthy of further study.

\section{Introduction}

Chronic diseases in young children have the potential to adversely affect parental quality of life. Intermittent exotropia is typically a chronic condition, and this condition may adversely affect parental quality of life.1 There are few data on the impact of childhood intermittent exotropia on parents.

The Intermittent Exotropia Quality of Life Questionnaire (IXTQ) is a newly developed and validated health-related quality of life (HRQOL) instrument that was designed for children with intermittent exotropia and their parents.2,3 The IXTQ consists of 3 components: (1) a

Reprint requests to: Dr. Jonathan Holmes, Ophthalmology W7, Mayo Clinic, Rochester MN, 55905 (holmes.jonathan@mayo.edu). Results from this study were presented as a poster at the annual meeting of the Association for Research in Vision and Ophthalmology, Ft. Lauderdale, Florida, May 2-6, 2010.

Publisher's Disclaimer: This is a PDF file of an unedited manuscript that has been accepted for publication. As a service to our customers we are providing this early version of the manuscript. The manuscript will undergo copyediting, typesetting, and review of the resulting proof before it is published in its final citable form. Please note that during the production process errors may be discovered which could affect the content, and all legal disclaimers that apply to the journal pertain. 
child self-report questionnaire to measure HRQOL for children as young as 5 years, (2) a proxy, parent-report questionnaire to measure child HRQOL for children as young as 2 years, and (3) a parent self-report questionnaire to measure parental HRQOL (Parent IXTQ).

The Pediatric Quality of Life Inventory Family Impact Module (PedsQL FIM) is a selfadministered HRQOL instrument designed to measure the impact of pediatric chronic health conditions on parents and the family, parent-reported family daily activities and family relationships.4 Since its development in 2004, the PedsQL FIM has been used to study the effects of childhood illness on parental HRQOL for chronic pain,5 kidney transplantation,6 and sickle cell disease.7

In the present study, we compared the HRQOL of parents of children with intermittent exotropia using the Parent IXTQ and the PedsQL FIM.

\section{Subjects and Methods}

Institutional review board approval was obtained and the parent of each child gave informed consent before participating. All procedures and data collection were conducted in a manner compliant with the Health Insurance Portability and Accountability Act.

We prospectively recruited one parent for each of 59 children with intermittent exotropia to complete the Parent IXTQ and PedsQL FIM; the parent was recruited during the child's eye examination at an outpatient clinic. Aside from intermittent exotropia, children had no other eye disease besides refractive error: 15 (25\%) habitually wore spectacles. Parents were required to be comfortably conversant in English to be included. The same parent completed both questionnaires. In 46 cases (78\%) the mother completed the questionnaires.

The median age of children was 7 years (range, 3 to 16 years). Median visual acuity (with spectacles, if needed) was 20/20 in each eye (range, 20/15-20/80, right eye; 20/15-20/100, left eye). Median angle of distance exodeviation measured by prism and alternate cover test was $25^{\Delta}$ (range, $0^{\Delta}-40^{\Delta}$ ) and median near exodeviation was $16^{\Delta}\left(\right.$ range, $\left.2^{\Delta_{-}} 40^{\Delta}\right)$. Control of the exodeviation was assessed using a previously described 6-point control scale8 at distance and at near. During a 30-second observation period, control was graded 5 for a constant exotropia, 4 for an exotropia present greater than $50 \%$ of the observation period, and 3 for an exotropia present less than 50\% of the observation period. If no exotropia was observed, control was rated following each of three 10-second dissociations (with the final score taken as the worst of the three): a control score of 2 indicated recovery of exodeviation in $>5$ seconds following dissociation; a control score of 1 , recovery in 1-5 seconds; a score of 0 , recovery in $<1$ second. In our cohort of 59 children with intermittent exotropia, median control scores were 2 (range, 0-5, quartiles 1 and 4 ) at distance fixation and 1 (range, 0-5, quartiles 0 and 1.5) at near. Parent IXTQ data from 12 of 59 subjects were included in a previous report describing the initial validation of the IXTQ.2

Twenty-nine parents of normal children (ie, without intermittent exotropia or other strabismus) were recruited to complete the Parent IXTQ and PedsQL FIM. The same parent completed both questionnaires. In 25 cases $(86 \%)$ the mother completed the questionnaires. The median age of children was 8 years (range, 5 to 13 years). Median visual acuity (with spectacles, if needed) was 20/20 in each eye (range, 20/15-20/30 in each eye). Sixteen (55\%) wore spectacles habitually. Parent IXTQ data from 21 of 29 normal subjects were included in a previous report 2 but we have not previously used these data to define normal threshold and have not previously compared data from an IXT cohort to those normal thresholds. 


\section{Questionnaires}

The Parent IXTQ is a questionnaire for parents comprised of 17 items in 3 subscales: function ( 8 items), psychosocial (7 items), and surgical ( 2 items) (available at http://public.pedig.jaeb.org, last accessed October 13, 2010). The PedsQL FIM is a questionnaire for parents comprised of 36 questions in 8 subscales: physical functioning (6 items), emotional functioning (5 items), social functioning (4 items), cognitive functioning (5 items), communication (3 items), worry (5 items), daily activities (3 items), and family relationships (5 items) (available for a fee at http://www.pedsql.org/index.html; limited-use version available at www.mapi-trust.org/questionnaires/accept/44, date last accessed October 13, 2010).

Both Parent IXTQ and PedsQL FIM use a 5-point Likert type scale for responses: Never (score 100), Almost never (score 75), Sometimes (score 50), Often (score 25), Almost always (score 0). Scores for each questionnaire (in its entirety and by subscale) were calculated for each subject by taking the mean of all responses for the entire questionnaire or for each subscale. Scores range from 0 (worst HRQOL) to 100 (best HRQOL).

\section{Analysis}

We compared median HRQOL scores between parents of normal children and parents of children with intermittent exotropia for both the Parent IXTQ and the PedsQL FIM using Wilcoxon rank sum tests.

Data from the normal cohort were used to calculate normal thresholds for each HRQOL instrument, which were defined as the Parent IXTQ and PedsQL FIM scores corresponding to the 5th percentile. Scores from a normal (or control) population will vary and have a distribution. One established method for defining a subnormal score is to take a threshold of the 5th percentile of a control population, where $95 \%$ of normal scores will fall above that threshold and only $5 \%$ of normal scores will fall below that threshold. For example, a score that equated to the 20th percentile of the normal (control) population would still be considered a "normal" score, but a score that equated to the 4th percentile of the normal (control) population would be considered subnormal. Using this nonparametric approach to estimating a normal range of values is especially important when the distribution of values does not follow a Gaussian distribution.9 We then compared the proportion of subnormal scores (ie, scores falling below the 5th percentile normal threshold) for the Parent IXTQ and PedsQL FIM in parents of children with intermittent exotropia using McNemar's tests. Proportions of subnormal scores on the Parent IXTQ and PedsQL FIM were compared between subgroups of younger ( 3 to 7 years old, $n=35$ ) and older ( 8 to 17 years old, $n=24$ ) children using Fisher exact tests.

\section{Results}

The median Parent IXTQ score was worse for parents of children with intermittent exotropia than for parents of visually normal children (70.6 vs 94.1, $p<0.0001)$, whereas the median PedsQL FIM scores between groups were similar (97.9 vs 95.8, $p=0.8$; Figure 1). All Parent IXTQ subscale scores (function, psychosocial, and surgical) showed worse median scores in parents of children with intermittent exotropia than in parents of normal children ( $p$ $<0.0001$ for each subscale), whereas all median PedsQL FIM subscale scores were similar; the worry subscale was the most affected PedsQL FIM subscale, although even the difference for that subscale did not reach statistical significance (Table 1).

Normal thresholds (5th percentile score) were calculated for the composite Parent IXTQ (58.8), composite PedsQL FIM (75.0) and for subscales (Table 2). Comparing proportions of subjects scoring below normal thresholds, more parents of children with intermittent 
exotropia scored below normal threshold with the Parent IXTQ than with the PedsQL FIM (31\% vs $12 \% ; p=0.008$; Figure 2 ).

Comparing younger to older children, the proportion of subnormal scores was similar for parents of younger children ( 3 to 7 years old, $\mathrm{n}=35$ ) and older children ( 8 to 17 years old, $\mathrm{n}$ $=24)$ on both the Parent IXTQ $(26 \%, 95 \%$ CI, $12 \%-43 \%$, vs 38\%, 95\% CI, 19\%-59\%; $p=$ 0.4 , difference of $12 \%, 95 \% \mathrm{CI},-12 \%$ to $36 \%)$, and on the PedsQL FIM (11\%, CI $3 \%-27 \%$, vs $13 \%$, CI $3 \%-33 \% ; p=1.0$; difference of $2 \%$, CI $-16 \%$ to $18 \%$ ).

Regarding subscales, the Parent IXTQ subscale with the highest proportion of subnormal scores was "function" (29\%, Table 2). The PedsQL FIM subscale with the highest proportion of subnormal scores was emotional functioning (15\%, Table 2 ).

\section{Discussion}

In the present study of parental HRQOL in childhood intermittent exotropia, HRQOL scores in parents of children with intermittent exotropia were worse than in parents of normal, nonstrabismic children using the Parent IXTQ, but not using the PedsQL FIM. Comparing the proportion of subnormal scores, a greater proportion of parents of children with intermittent exotropia scored below normal threshold using the Parent IXTQ than using the PedsQL FIM.

There is very little literature reporting the impact of childhood strabismus on parental HRQOL. A study by Akay and colleagues 10 found that mothers of children with strabismus had more distress, dissatisfaction, and poorer family relationships than mothers of children without strabismus. Nevertheless, the majority of children in Akay and colleagues' cohort had esotropia, and it is possible that parents of children with intermittent exotropia are not as severely affected since children with intermittent exotropia frequently have good visual acuity, more normal appearance, good stereoacuity, and many may remain stable without treatment.11 Yet the present study suggests that parents of children with intermittent exotropia do worry about the potential effects this condition may have on their child. Parental concerns may play a role in the decision to intervene in childhood intermittent exotropia, and knowledge of these parental concerns is important for the treating provider. The Parent IXTQ is an instrument that may be used in quantifying these potential parental HRQOL concerns.

We used an established, generic HRQOL instrument and an IXT-specific HRQOL instrument to assess parental HRQOL in the present study. It is possible that the Parent IXTQ and PedsQL FIM may measure different aspects of parental HRQOL. A previous study by Hatt and colleagues1 found that worry is frequently expressed by parents of children with intermittent exotropia and the design of the Parent IXTQ was based on these findings. 2 In contrast the PedsQL FIM measures a wide range of potential parental HRQOL concerns (eg, physical, emotional, social, and cognitive functioning, communication, worry, daily activities, relationships), of which worry is only a small component: only 5 of 36 questions address worry in the PedsQL FIM. Interestingly, the most affected subscale in the PedsQL FIM was the worry subscale (Table 1). Overall, childhood intermittent exotropia may only affect very specific aspects of parental HRQOL (eg, parental worry about their child's eyes) which may not be measurable by the generic PedsQL FIM.

One limitation of the present study was homogeneity of our sample. It is possible that different cohorts (eg, those of different races or socioeconomic backgrounds) may give different HRQOL responses with either Parent IXTQ or PedsQL FIM. It is also possible that parents of children with even more severe intermittent exotropia might have responded differently, nevertheless our cohort of children with intermittent exotropia included a wide 
spectrum of severity. Another potential limitation was the difference in proportions of children in each group wearing spectacles, because spectacle wear alone affects parental health-related quality of life, when measured with the Parent IXTQ.12 Nevertheless, the intermittent exotropia cohort in the present study had a lower proportion of spectacle wear than the visually normal cohort, which would potentially bias against finding an effect of intermittent exotropia on Parental HRQOL, but we still found an effect.In addition, our normal threshold may have been slightly depressed by a spectacle effect, resulting in a lower proportion of parents of children with intermittent exotropia reporting subnormal HRQOL. Nevertheless we still found a higher proportion of subnormal scores on the IXTQ.

In conclusion, the Parent IXTQ instrument detects subnormal parental HRQOL in a greater proportion of parents than the PedsQL FIM. Parental concerns may influence management of children with intermittent exotropia, and therefore the Parent IXTQ may be helpful in formally assessing parental HRQOL in families that have children with intermittent exotropia. The Parent IXTQ would help identify parents who are particularly worried about their child with intermittent exotropia, even in the face of mild disease and no evidence of deterioration. The treating physician would then be better able to counsel these parents, and avoid surgery when the primary driver is solely parental concern.

\section{Acknowledgments}

Financial support: Supported by National Institutes of Health Grants EY015799 (JMH), EY018810 (JMH), Research to Prevent Blindness, New York, NY (JMH as Olga Keith Weiss Scholar and an unrestricted grant to the Department of Ophthalmology, Mayo Clinic), and Mayo Foundation, Rochester, MN. None of the sponsors or funding organizations had a role in the design or conduct of this research. No authors have any financial/conflicting interests to disclose.

\section{References}

1. Hatt SR, Leske DA, Adams WE, Kirgis PA, Bradley EA, Holmes JM. Quality of life in intermittent exotropia: child and parent concerns. Arch Ophthalmol. 2008; 126:1525-9. [PubMed: 19001219]

2. Hatt SR, Leske DA, Yamada T, Bradley EA, Cole SR, Holmes JM. Development and initial validation of quality of life questionnaires for intermittent exotropia. Ophthalmology. 2010; 117:163-8. [PubMed: 19896195]

3. Hatt SR, Leske DA, Holmes JM. Comparison of quality-of-life instruments in childhood intermittent exotropia. J AAPOS. 2010; 14:221-6. [PubMed: 20417138]

4. Varni JW, Sherman SA, Burwinkle TM, Dickinson PE, Dixon P. The PedsQL Family Impact Module: preliminary reliability and validity. Health Qual Life Outcomes. 2004; 2:55. [PubMed: 15450120]

5. Mano KE, Khan KA, Ladwig RJ, Weisman SJ. The impact of pediatric chronic pain on parents' health-related quality of life and family functioning: Reliability and validity of the PedsQL 4.0 Family Impact Module. J Pediatr Psychol. 200910.1093/jpepsy/jsp099

6. Anthony SJ, Hebert D, Todd L, Korus M, Langlois V, Pool R, et al. Child and parental perspectives of multidimensional quality of life outcomes after kidney transplantation. Pediatr Transplant. 2010; 14:249-56. [PubMed: 19686446]

7. Panepinto JA, Hoffmann RG, Pajewski NM. A psychometric evaluation of the PedsQL Family Impact Module in parents of children with sickle cell disease. Health Qual Life Outcomes. 2009; 7:32. [PubMed: 19371442]

8. Mohney BG, Holmes JM. An office-based scale for assessing control in intermittent exotropia. Strabismus. 2006; 14:147-50. [PubMed: 16950743]

9. Reed AH, Henry RJ, Mason WB. Influence of statistical method used on the resulting estimate of normal range. Clin Chem. 1971; 17:275-84. [PubMed: 5552364]

10. Akay AP, Cakaloz B, Berk AT, Pasa E. Psychosocial aspects of mothers of children with strabismus. J AAPOS. 2005; 9:268-73. [PubMed: 15956948] 
11. Romanchuk KG, Dotchin SA, Zurevinsky J. The natural history of surgically untreated intermittent exotropia-looking into the distant future. J AAPOS. 2006; 10:225-31. [PubMed: 16814175]

12. Yamada T, Leske DA, Hatt SR, Holmes JM. Spectacle wear in children reduces parental healthrelated quality of life. J AAPOS. 2011; 15:000-000. 


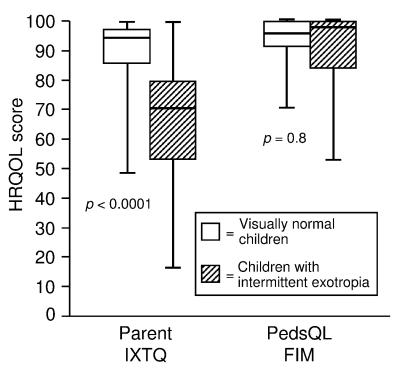

FIG 1.

Median Parent IXTQ scores were worse for parents of children with intermittent exotropia than parents of visually normal children (70.6 vs 94.1, $p<0.0001$ ), in contrast to PedsQL FIM scores (97.9 vs 95.8, $p=0.8)$. Higher scores indicate better health-related quality of life. Box boundaries indicate 1st quartile, median, and 3rd quartile values; whiskers indicate extreme values. 


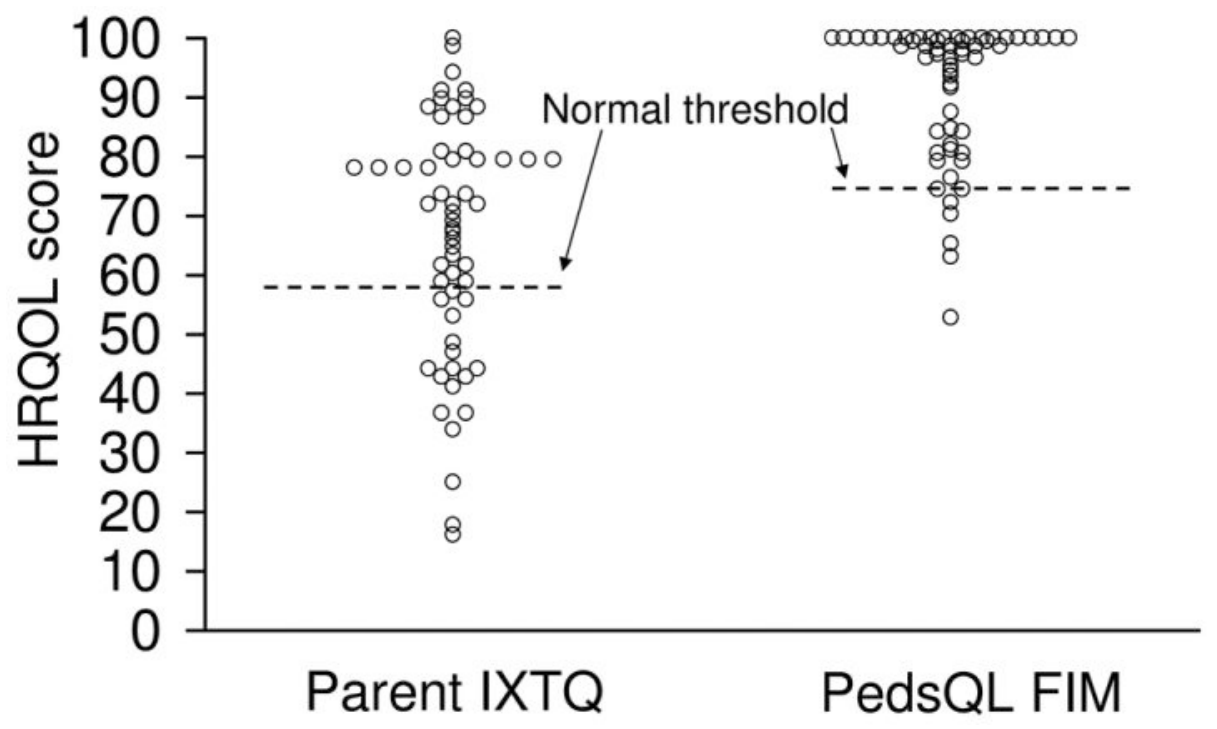

FIG 2.

Individual Parent IXTQ and PedsQL FIM scores compared to normal thresholds. A larger proportion of parents of children with intermittent exotropia scored subnormal on the Parent IXTQ versus PedsQL FIM (31\% vs $12 \%, p=0.008)$. Higher scores indicate better healthrelated quality of life. 


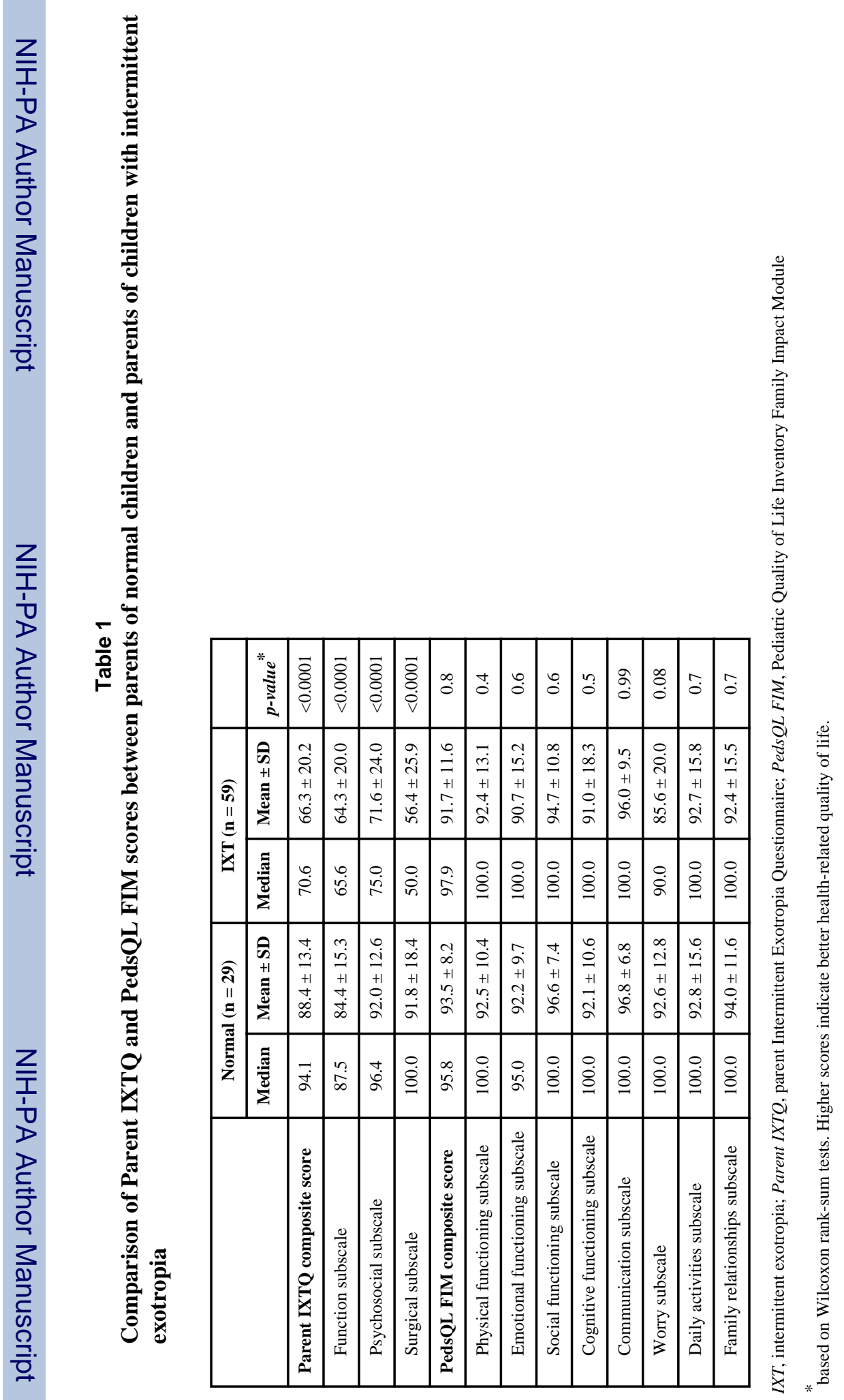


Table 2

Normal thresholds for Parent IXTQ and PedsQL FIM composite and subscale scores and proportion of intermittent exotropia (IXT) cohort who had subnormal scores

\begin{tabular}{|l|l|l|}
\hline & Normal threshold score & Proportion of IXT cohort scoring below normal threshold score \\
\hline Parent IXTQ composite score & 58.8 & $31 \%$ \\
\hline Function subscale & 53.1 & $29 \%$ \\
\hline Psychosocial subscale & 60.7 & $22 \%$ \\
\hline Surgical subscale & 37.5 & $19 \%$ \\
\hline PedsQL FIM composite score & 75.0 & $12 \%$ \\
\hline Physical functioning & 70.8 & $10 \%$ \\
\hline Emotional functioning & 75.0 & $15 \%$ \\
\hline Social functioning & 75.0 & $7 \%$ \\
\hline Cognitive functioning & 75.0 & $14 \%$ \\
\hline Communication & 83.3 & $8 \%$ \\
\hline Worry & 65.0 & $12 \%$ \\
\hline Daily activities & 58.3 & $5 \%$ \\
\hline Family relationships & 70.0 & $12 \%$ \\
\hline
\end{tabular}

IXT, intermittent exotropia; Parent IXTQ, parent Intermittent Exotropia Questionnaire; PedsQL FIM, Pediatric Quality of Life Inventory Family Impact Module

* Derived from visually normal children without strabismus 